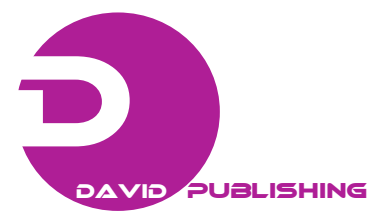

\title{
iRobot Create Used in Education
}

\author{
Martin Dekan, František Duchoň, Ladislav Jurišica, Anton Vitko and Andrej Babinec \\ Institute of Control and Industrial Informatics, Faculty of Electrical Engineering and Information Technology, Slovak University of \\ Technology in Bratislava, Bratislava 812 19, Slovakia
}

Received: January 11, 2013 / Accepted: February 7, 2013 / Published: April 25, 2013.

\begin{abstract}
There are many robotic platforms usable in the educational process. In the introduction, some of the educational platforms are presented. The main body of this paper deals with educational robotic platform iRobot Create. In this paper, main parts of this platform are described, such as mechanics, sensors and actuators. The software equipment with communication protocol of this platform is also described. The results of the analysis of this platform show its rich possibilities in its use in educational process such as mechanics, electronics and algorithms used in robotics.
\end{abstract}

Key words: Mobile robot, educational platform, iRobot Create.

\section{Introduction}

Service robots for the educational process serve as motivation for the future generations of researchers or as systems deployed in the real world, which demonstrate the state of the problem in robotics. The purpose of systems deployed in the real world is human interaction with robots above all. In other words, such robots provide entertainment or therapeutic recreation. The operation of such systems does not require specialists educated in this field. It turns out that the teaching of programming in programming languages is much more interesting for the students if the result is connected to real life situations. In contrast, programming of abstract functions (e.g., recursive function for the Taylor series) is discouraging. Another aspect of using robots in education is education itself. The robot can be used in teaching the mechatronics and related fields of science, because it is a typical mechatronic system. Robots can be used as teaching aids in such fields as science, technology and engineering. In addition to this, the students learn to co-operate in team, identify and solve problems as well as to understand new technologies. All in all we can say

Corresponding author: František Duchoň, Assoc. Prof., research field: robotics. E-mail: frantisek.duchon@stuba.sk. that teaching robotics can raise comprehensive engineers. Surprisingly, women are more attracted to the technical disciplines thanks to robots. Another aspect of the use of robots in education is that the robot can serve as a teacher's assistant. In this case, the robot stands for whole season companion, assistant and even as an intellectual enhancement. The robots with social skills are suitable for such tasks and it seems that this area has great potential for at least two reasons. The first reason is its novelty and it can increase enthusiasm of students for learning. The second reason is the unpredictable behavior of such robot. Such behavior can be useful in therapeutic situations. Anyway, the interaction with robot is a means of exploring and takes a different way of the traditional more or less rigid teaching methods, therefore it may be a major motivating factor in the learning of students [1-2].

This paper is organized as follows: Section 2 describes existing robotic platform used in education; sections 3 and 4 introduce iRobot Create platform and describe in detail its possibilities in educational process; section 5 concludes the paper.

\section{Educational Platforms}

Robotic platforms for the educational purpose can be 
divided into two categories:

- Research platforms - expensive, precise platform designed to promote research in robotics and related industries;

- Cheap robotic kits - exist in large quantities and are intended for schools and hobbyists.

The robot Hero-1, sold as a cheap kit, was among the first robotic platforms for education. Although detailed information on folding of the construction was available, information on the principle of operation was not available in the case of this robot. With the advent of personal robotics and lack of additional value to learning of similar types of robots, these types of robots have disappeared from the market. Meanwhile, there are many companies involved in designing the robots for the purposes of education. As an example, let us mention the K-Team and their well-known Khepera robot, which is designed for education at high schools and universities.

The most famous robotic kit is the Lego Mindstorm. Some components from the Lego Company have been part of some of the robots for a long time, though the real passion for robotics followed the arrival of its own control system and its own sensors. Another well-known platform is the E-puck, whose diameter is only $70 \mathrm{~mm}$. Three accelerometers, proximity sensors, three microphones, a color camera, a number of actuators, Bluetooth communication and dsPIC processor are implemented in such small diameter. The Garcia robot from the company Acroname is also proportionally small robot $(25 \mathrm{~cm}, 18 \mathrm{~cm}, 10 \mathrm{~cm})$, which contains a small number of sensors and a manipulator [3].

The most famous humanoid robot used in education is the Robosapien. Although this is just a remote controlled toy, some enthusiasts managed to make accessible the robot control system by using reverse engineering. This makes possible to use the robot as a fully functional programmable robotic platform. Nowadays, the best known service robot is probably the robotic vacuum cleaner Roomba from the iRobot company. This robot was not originally meant as an educational robotic platform, but as with the Robosapien, some enthusiasts managed to access its control system. The iRobot responded to these attempts and created an educational platform iRobot Create on which it is possible to connect various sensors and add a custom control system. Researchers and enthusiasts from all over the world have proved that on this platform it is possible to construct almost anything from musical instruments to robots for surveillance. In addition, many schools and universities use this platform in the educational process [1].

\section{Platform iRobot Create-Hardware}

As mentioned above, the iRobot Create (Figs. 1-2) is based on the same grounds as the robotic vacuum cleaner Roomba, so it has similar hardware equipment. It is a four-wheel differential drive chassis with two driving wheels that contain incremental encoder and sensor of lifting, one supporting swing wheel with a sensor of lifting and one supporting fixed wheel [4].

To navigate in the area Create has a distance sensor, a distance sensor designed to avoid stairs and bump sensors hidden in the front bumper. On the bumper, there is also an omnidirectional IR receiver that receives information from the docking station, virtual walls and remote control. Of course there are also sensor of internal parameters such as battery status, current consumption and status of buttons [4-5].

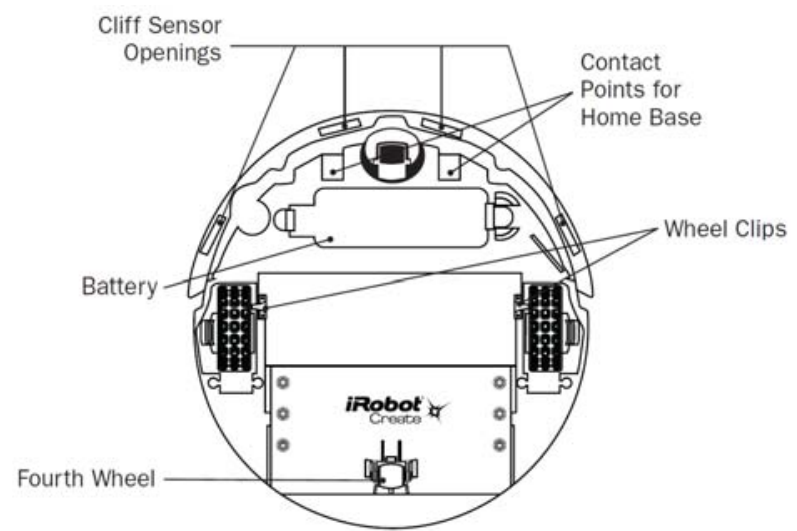

Fig. 1 Chassis iRobot Create with highlighted components [4]. 


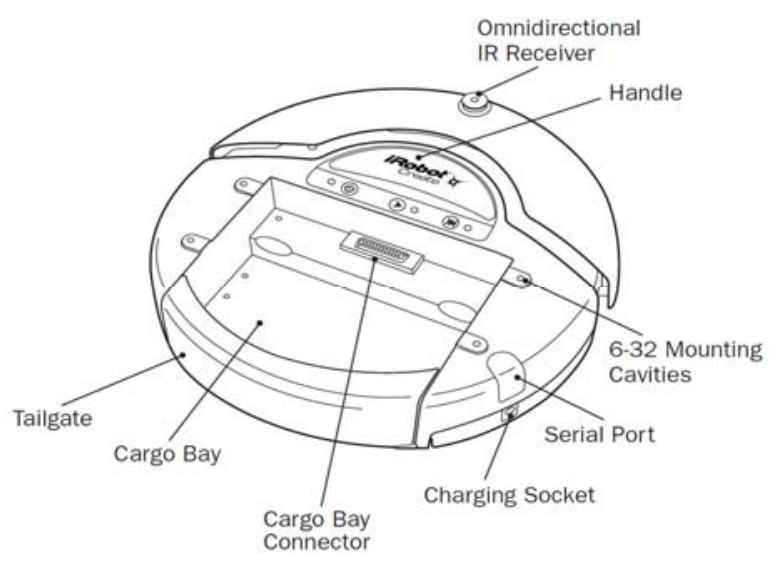

Fig. 2 iRobot Create [4].

Communication and control of the iRobot Create chassis can be conducted by multiple interfaces. There is a 25-pin port (Fig. 3 and Table 1) in the storage space on which you can attach Command module (Fig. 4) or Bluetooth Adapter Module, or custom control can be attached as well [7]. Another way to control functions of iRobot Create is a serial port that can be linked to a computer by a cable (Fig. 3 and Table 2) [4-5].

As already mentioned, custom analogous or digital sensors can be directly attached to the iRobot Create cargo bay connector or can be plugged into one of the four ePortal located in the Command Module [6, 8].

\section{Platform iRobot Create-Software}

Open interface protocol is implemented in the iRobot Create platform to control the robot's functions and obtain information from the sensors. This protocol operates on the principle of short byte messages, where the first byte determines the message type and the following bytes are used for data. Sample open interface protocol is in Table 3 [5].

The advantage of open interface protocol is the ability to create a simple script to control the robot motion without the use of programming resources. Another advantage of this protocol is undoubtedly the possibility to define which data from memory are to be sent from Create, which simplifies and speeds up the data processing. Individual sensoric data and its belonging to packets are shown in Table 4 [5].

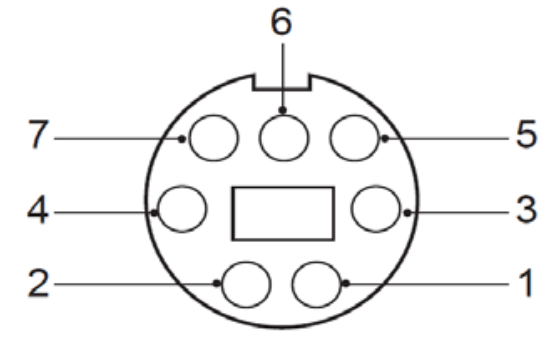

(13) (12) (11) (10) (9) (8) (7) (6) (5) (3) (2) (1) (25) (24) (23) (22) (21) (20) (17) (17) (17) (16) (15) (14)

Fig. 3 Serial connector (left) and the cargo bay connector (right) [5].

Table 1 Types of Cargo bay connector pins [5].

\begin{tabular}{|c|c|c|}
\hline Pin & Name & Description \\
\hline 1 & Rxd & 0-5 V Serial input to iRobot Create \\
\hline 2 & Txd & $\begin{array}{l}0-5 \text { V Serial output from iRobot } \\
\text { Create }\end{array}$ \\
\hline 3 & $\begin{array}{l}\text { Power control } \\
\text { toggle }\end{array}$ & $\begin{array}{l}0-5 \text { V Serial output from iRobot } \\
\text { Create }\end{array}$ \\
\hline 4 & Analog input & $0-5 \mathrm{~V}$ analog input to iRobot Create \\
\hline 5 & Digital input 1 & 0-5 V digital input to iRobot Create \\
\hline 6 & Digital input 3 & 0-5 V digital input to iRobot Create \\
\hline 7 & Digital output 1 & $\begin{array}{l}0-5 \mathrm{~V}, 20 \mathrm{~mA} \text { digital output from } \\
\text { iRobot Create }\end{array}$ \\
\hline 8 & Switched 5V & $\begin{array}{l}\text { Provides a regulated } 5 \mathrm{~V} 100 \mathrm{~mA} \\
\text { supply and analogue reference voltage } \\
\text { when iRobot Create is switched on }\end{array}$ \\
\hline 9 & Vpwr & $\begin{array}{l}\text { iRobot Create battery voltage } \\
\text { (unregulated), } 0.5 \mathrm{~A}\end{array}$ \\
\hline 10 & Switched Vpwr & $\begin{array}{l}\text { Provides battery power @ } 1.5 \text { A when } \\
\text { iRobot Create is powered on. }\end{array}$ \\
\hline 11 & Switched Vpwr & $\begin{array}{l}\text { Provides battery power@1.5 A when } \\
\text { iRobot Create is powered on. }\end{array}$ \\
\hline 12 & Switched Vpwr & $\begin{array}{l}\text { Provides battery power@1.5 A when } \\
\text { iRobot Create is powered on. }\end{array}$ \\
\hline 13 & Robot charging & $\begin{array}{l}\text { Provides battery power@1.5 A when } \\
\text { iRobot Create is powered on. }\end{array}$ \\
\hline 14 & GND & iRobot Create battery ground \\
\hline 15 & $\mathrm{BRC}$ & $\begin{array}{l}0-5 \mathrm{~V} \text { digital input to iRobot Create } \\
\text { which can also be used to change the } \\
\text { baud rate to } 19200 \text { (see below) }\end{array}$ \\
\hline 16 & GND & iRobot Create battery ground \\
\hline 17 & Digital input 0 & 0-5 V digital input to iRobot Create \\
\hline 18 & Digital input 2 & 0-5 V digital input to iRobot Create \\
\hline 19 & Digital output 0 & $\begin{array}{l}0-5 \mathrm{~V}, 20 \mathrm{~mA} \text { digital output from } \\
\text { iRobot Create }\end{array}$ \\
\hline 20 & Digital output 2 & $\begin{array}{l}0-5 \mathrm{~V}, 20 \mathrm{~mA} \text { digital output from } \\
\text { iRobot Create }\end{array}$ \\
\hline 21 & GND & iRobot Create battery ground \\
\hline 22 & Low side driver 0 & 0.5 A low side driver from Create \\
\hline 23 & Low side driver 1 & 0.5 A low side driver from Create \\
\hline 24 & Low side driver 2 & 1.5 A low side driver from Create \\
\hline 25 & GND & iRobot Create battery ground \\
\hline
\end{tabular}




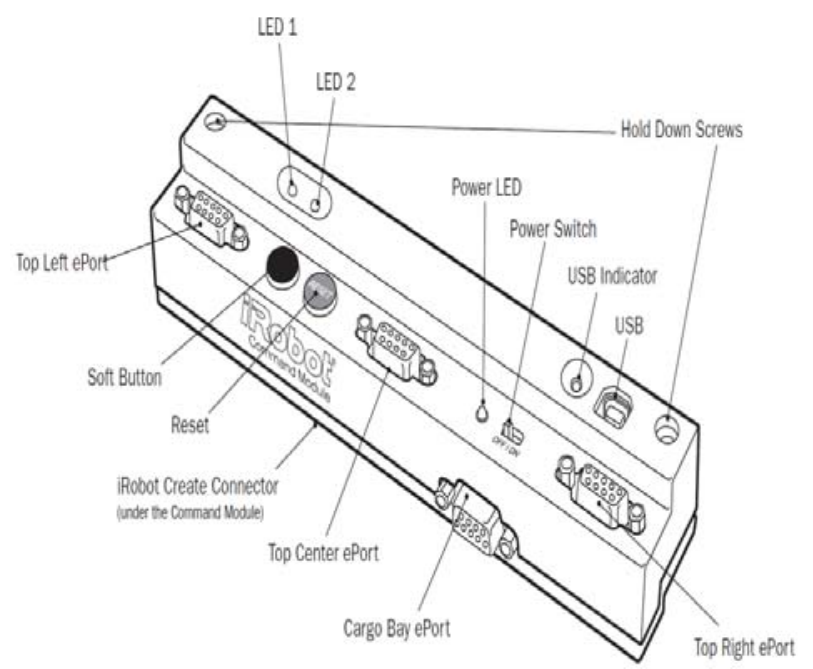

Fig. 4 Command module [6].
Table 2 Types of serial connector pins [5].

\begin{tabular}{lll}
\hline Pin & Name & Description \\
\hline 1 & Vpwr & Create battery + (unregulated) \\
2 & Vpwr & Create battery + (unregulated) \\
3 & Rxd & 0-5 V Serial input to Create \\
4 & Txd & 0-5 V Serial output from Create \\
5 & BRC & Baud Rate Change \\
6 & GND & Create battery ground \\
7 & GND & Create battery ground \\
\hline
\end{tabular}

Table 3 Sample open interface protocol [5].

\begin{tabular}{lcccc}
\hline Command Opcode & $\begin{array}{l}\text { Data } \\
\text { Bytes 1 }\end{array}$ & $\begin{array}{l}\text { Data } \\
\text { Bytes 2 }\end{array}$ & $\begin{array}{l}\text { Data } \\
\text { Bytes 3 }\end{array}$ & $\begin{array}{l}\text { Data } \\
\text { Bytes 4 }\end{array}$ \\
\hline Drive & 137 & $\begin{array}{l}\text { Velocity } \\
(-500-500) \mathrm{mm} / \mathrm{s}\end{array}$ & $\begin{array}{c}\text { Radius } \\
(-2000-2000) \mathrm{mm}\end{array}$ \\
Sensors & 142 & $\begin{array}{l}\text { Packet } \\
\text { ID (0-42) }\end{array}$ & \\
\hline
\end{tabular}

Table 4 Data provided by iRobot Create and their affiliation to the packets [5].

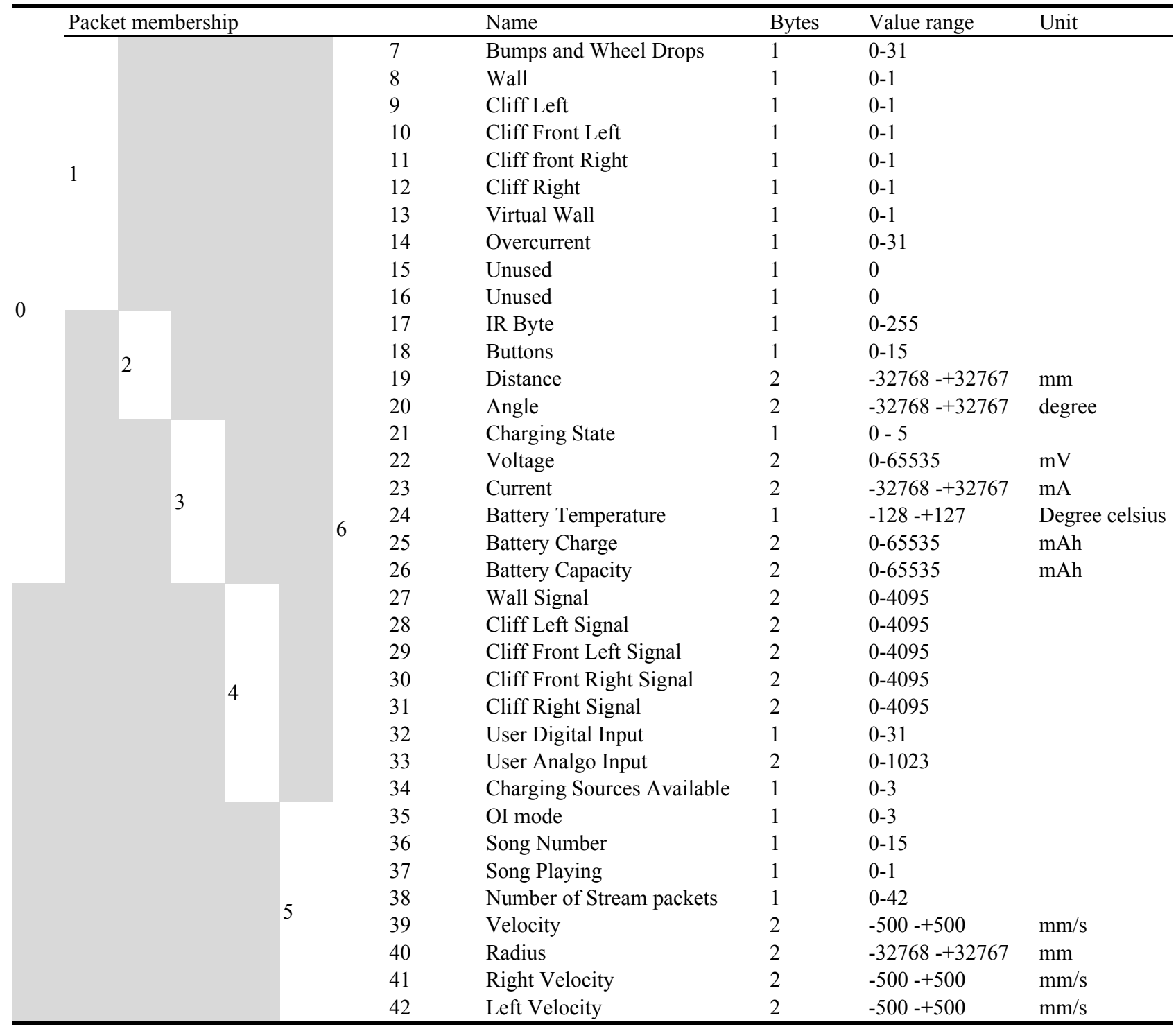


For instance, we can demonstrate Packet number 17 which determines the state of the infrared sensor. Information is encoded into one byte from the range of values from 0 to 255 and stores information on IR signals sent by remote control, base, using robots Crate Send IR commands and other devices created by the user. Values of the byte and their explanation are shown in Table 5.

As shown in Table 5, to identify the location of the base iRobot Create uses three information, their purpose and distribution in space are shown in Fig. 5.

There are several possibilities to program the movement and function of the iRobot Create. One way is to link the iRobot Create with a computer either via serial line or wireless communication module. The advantage of this solution is the possibility of using the processing power of computer to calculate algorithms for which the processing power of Create is not sufficient. Another option is to control the iRobot Create from the Command Module. This module hides ATmega microprocessor on which the program in $\mathrm{C}$ or Assembler can be recorded. Inputs and outputs of the microprocessor are connected to the port ePORT which can be used to connect other devices.

iRobot company support their products with hardware and code samples that are very simple and makes the programmer in a vivid manner familiar with the basic principles of programming and offer many pre-made functions usable in other projects. Small sample of the source code implementing the robot motion is shown in Fig. 6. It is possible to control all functions of a mobile chassis iRobot Create in a similar manner.

\section{Conclusions}

From the previous sections, which dealt with the software and hardware of the robotics platform, iRobot Create results numerous tasks that can be solved on the platform be assigned to the students of robotics. On this platform, students can learn the basic principle of controlling the mobile robots, sensor systems, hardware
Table 5 Importance of values for IR data bytes packet [5].

\begin{tabular}{|c|c|c|}
\hline $\begin{array}{l}\text { Sent by iRobot } \\
\text { device }\end{array}$ & $\begin{array}{l}\text { Character } \\
\text { value }\end{array}$ & Character name \\
\hline \multirow{13}{*}{$\begin{array}{l}\text { Remote } \\
\text { Control }\end{array}$} & 129 & Left \\
\hline & 130 & Forward \\
\hline & 131 & Right \\
\hline & 132 & Spot \\
\hline & 133 & Max \\
\hline & 134 & Small \\
\hline & 135 & Medium \\
\hline & 136 & Large/Clean \\
\hline & 137 & Pause \\
\hline & 138 & Power \\
\hline & 139 & Arc-forward-left \\
\hline & 140 & Arc-forward-right \\
\hline & 141 & Drive-stop \\
\hline Scheduling & 142 & Send All \\
\hline Remote & 143 & Seek Dock \\
\hline \multirow{8}{*}{ Home Base } & 240 & Reserved \\
\hline & 248 & Red Buoy \\
\hline & 244 & Green Buoy \\
\hline & 242 & Force Field \\
\hline & 252 & Red Buoy and Green Buoy \\
\hline & 250 & Red Buoy and Force Field \\
\hline & 246 & Green Buoy and Force Field \\
\hline & 254 & $\begin{array}{l}\text { Red Buoy and Green Buoy and } \\
\text { Force field }\end{array}$ \\
\hline
\end{tabular}

Fig. 5 Distribution of base identifiers [5].

design, algorithms, and other less general areas. Students have the opportunity to learn how to control the robot autonomously or remotely. By the solutions of the remote control, it can be shown the impact of the interface operator-robot and the communication module for such control. Various methods of localization and navigation can be demonstrated on autonomous control of mobile robot. Moreover, this exercise can be extended to hardware design of their 


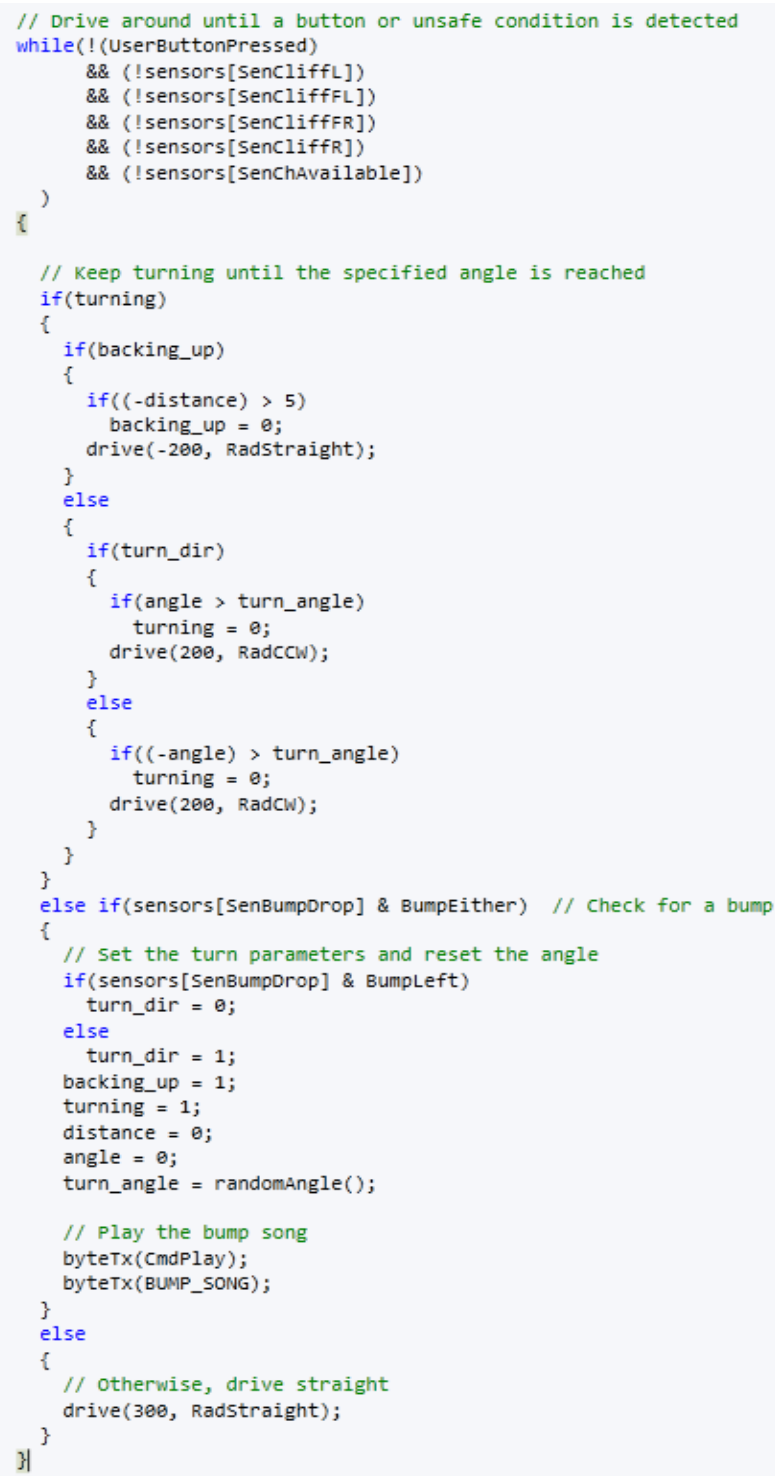

Fig. 6 Sample source code. own sensor systems and the dynamic obstacle avoidance. Thanks to the low price of this platform it can also be used to educate students in multi-agent control. iRobot Create platform is impressive and effective platform in the educational process. The proof to this is the deployment of this robotic platform in the subject mobile robotic systems at URPI FEI STU.

\section{Acknowledgment}

This work was supported by MS SR under contract APVV-0539-11 and VEGA 1/0177/11.

\section{References}

[1] B. Siciliano, O. Khatib, Handbook of Robotics, Springer, 2008.

[2] M. Blaho, M. Foltin, P. Fodrek, J. Murgaš, Students perspective on improving programming courses, International Journal of Education and Information Technologies 6 (1) (2012) 17-24.

[3] R. Balogh, Acrob-An educational robotic platform, AT\&P Journal PLUS 2 (2010) 6-9.

[4] iRobot@ Create Owner's guide, www.irobot.com.

[5] iRobot ${ }^{\circledR}$ Create OPEN INTERFACE, www.irobot.com.

[6] iRobot ${ }^{\circledR}$ Command Module Owner's manual, www.irobot.com.

[7] J. Rodina, P. Hubinský, Stabilization of the segway like mobile platform by using MEMS sensors, in: Proceedings of 3rd International Conference on Modelling of the Mechanics and Mechatronics Systems (MMaMS'2009), 2009, pp. 402-406.

[8] M. Kelemen, Components and modules of mechatronic systems, 1st ed., TU, Košice, 2004. 